\title{
Bases epidemiológicas para análise das más oclusões morfológicas como fatores de risco no desenvol- vimento das desordens temporomandibulares de origem articular
}

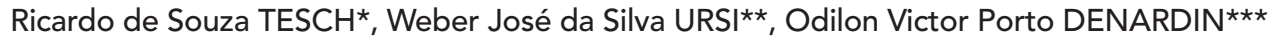

\begin{abstract}
Resumo
Dentre as pesquisas empreendidas no campo da epidemiologia, um grupo específico aborda patologias de etiologia desconhecida ou não totalmente compreendidas. É dentro deste grupo que estão situadas as desordens temporomandibulares (DTM). Três estratégias observacionais básicas têm sido utilizadas para abordar o papel etiológico da má oclusão no desenvolvimento das DTM, dentro do repertório epidemiológico. São elas: estudos do tipo transversal, estudos de caso controle e estudos de coorte. Alguns experimentos clínicos são realizados com base na remoção do fator etiológico suspeito. Com base em uma revisão estruturada da literatura, a partir da metodologia empregada nos estudos selecionados, podemos concluir que a definição dos possíveis fatores etiológicos relacionados a subgrupos específicos de DTM é fundamental para que o papel das más oclusões no desenvolvimento destas desordens, embora pareça pequeno quando baseado nas evidências disponíveis, não seja subestimado. Pode ser útil a caracterização de uma oclusão normal como aquela associada como o menor risco para o desenvolvimento de problemas de DTM, mas é provavelmente inapropriada a aplicação destes parâmetros para reverter um problema intra-capsular já estabelecido. $\mathrm{O}$ conceito de uma oclusão de baixo fator de risco implicaria em um pequeno desvio entre RC e MIH, pequeno transpasse horizontal, transpasse vertical positivo e ausência de mordida cruzada posterior. Este conceito é compatível com o conceito de oclusão normal defendido por décadas, embora uma variação do normal ao invés de um critério absoluto deva ser permitida. Embora provavelmente seja prudente estabelecer metas morfológicas terapêuticas que busquem o que é observado em oclusões não tratadas julgadas normais ou ideais, o estabelecimento de uma oclusão que alcance todos os critérios gnatológicos, por meio de tratamento ortodôntico, talvez seja impossível e provavelmente desnecessário.
\end{abstract}

Palavras-chave: Má oclusão. Desordens temporomandibulares. Epidemiologia.

* Mestre em Ciências da Saúde pelo Hosphel-SP. Especialista em Ortodontia pela ACDC-Campinas. Professor do Curso de Especialização em Ortodontia da ABO-RJ - Petrópolis. Chefe do Serviço de DTM e Dor Orofacial da Faculdade de Medicina de Petrópolis.

** Mestre e Doutor em Ortodontia pela FOB-USP. Professor Dr. UNESP - São José dos Campos. Coordenador do Curso de Especialização em Ortodontia da APCD- São José dos Campos.

*** Doutor em Medicina pela EPM-Unifesp. Professor Responsável pelas Disciplinas de Epidemiologia Clínica e Bioestatística do Curso de Pós-Graduação em Ciências da Saúde do Hosphel-SP. 


\section{INTRODUÇÃO E REVISÃO DE LITERATURA}

O termo epidemiologia pode ser definido como o "estudo dos fatores que determinam a freqüência e a distribuição das doenças nas coletividades humanas"2. A epidemiologia moderna está situada no limiar de um fascinante campo de novos questionamentos sobre o processo saúde-doença e sua relação com a sociedade. Dentre as pesquisas empreendidas neste campo, um grupo específico aborda patologias de etiologia desconhecida ou não totalmente compreendidas. É dentro deste grupo que estão situadas as desordens temporomandibulares (DTM), as quais podem ser definidas como um grupo de condições dolorosas ou não funcionais, envolvendo os músculos da mastigação e/ou a articulação temporomandibular (ATM) ${ }^{7}$.

O problema epidemiológico é o produto das alterações ocorridas no sistema população - agentes etiológicos - ambiente, sendo estas alterações possíveis fatores responsáveis, ou de risco, para a produção de doenças ${ }^{2}$. No decorrer de toda a história da ciência ocidental, o desenvolvimento da biologia caminhou de mãos dadas com o da área médica. Assim sendo, é natural que a concepção biológica mecanicista da vida, fundamentada no paradigma cartesiano, dominasse também a atitude dos profissionais de saúde em relação à saúde e à doença ${ }^{5}$. Daí a necessidade histórica de se tentar atribuir a apenas uma parte do organismo todas as causas de problemas de origem, freqüentemente, multifatoriais. Assim, o modelo multifatorial da etiologia das DTM foi por muito tempo ignorado e estas explicadas por inúmeras hipóteses unifatoriais não totalmente testadas em sua veracidade.

A formulação de hipóteses é etapa indispensável de qualquer pesquisa que se pretenda científica. Podem ser definidas como conjecturas com as quais se procura explicar por tentativa, fenômenos ocorridos ou ocorrentes e serão científicas à medida que afirmarem relações entre variáveis e forem abertas à refutação². Muitas das hipóteses formuladas na atividade acadêmica para responder a questionamentos à cerca da etiologia das doenças são levantadas pela associação das visões clínica e epidemiológica.

No que se refere às DTM, a formulação de um pressuposto teórico, baseado em um ideal morfológico e funcional, difundido pela crescente influência e desenvolvimento das escolas de oclusão, depositou nas más oclusões um papel prioritário no desenvolvimento destas patologias. Estes conceitos foram sedimentados pelo sucesso relativo das terapias oclusais, sejam estas reversíveis ou irreversíveis. Contudo, a relação entre estas variáveis necessita de confirmação científica.

Embora o conhecimento científico a respeito dos inúmeros aspectos referentes à etiologia das DTM tenha avançado de maneira significante nas últimas décadas, ainda existe uma grande lacuna entre o diagnóstico e terapêutica baseados em evidências científicas e a prática clínica. Isto se deve, em parte, às dificuldades enfrentadas pelo clínico em reconhecer, dentre o grande volume de literatura publicada, quais estudos são válidos e podem ser aplicáveis às reais necessidades do paciente.

Tais estudos devem definir com a maior precisão possível qual a estratégia de ação mais adequada aos objetivos da pesquisa. Três estratégias observacionais básicas têm sido utilizadas para abordar o papel etiológico da oclusão no desenvolvimento das DTM, dentro do repertório epidemiológico. São elas: estudos do tipo transversal, estudos de caso controle e estudos de coorte. Dificilmente encontramos estudos executados com a inclusão e o controle do fator suspeito em grupos experimentais, devido a envolvimentos éticos óbvios. Contudo, alguns experimentos clínicos são realizados com base na remoção do fator etiológico suspeito.

\section{CONSIDERAÇÕES GERAIS SOBRE EPIDEMIOLOGIA ANALÍTICA}

A associação entre o fator que é supostamente etiológico para uma patologia e esta pode ser estabelecida sob o ponto de vista populacional. Isto 
significa que ao se verificar a possibilidade deste relacionamento, a população em foco poderá ser dividia em quatro grupos, sendo estes: o grupo dos atingidos, o dos não atingidos, o grupo dos expostos e o dos não expostos. Os dois primeiros são relativos às patologias em questão, como por exemplo as DTM, e os dois últimos ao fator etiológico que supostamente lhes é associado, como por exemplo a presença de má oclusão. Assim sendo, podemos estabelecer relações estatísticas visando o conhecimento epidemiológico (Tab. 1).

Objetiva-se, portanto, saber se a proporção dos que, simultaneamente, apresentam o fator etiológico e a patologia (atingidos + expostos) é significativamente maior ou diferente do que seria de se esperar se estes dois eventos não fossem relacionados entre si.

Há duas maneiras de respondermos a esta questão. Uma estabelece grupos de expostos e não expostos e, seguindo-os ao longo do tempo, compara-se à freqüência da ocorrência da patologia entre os dois grupos. Estes estudos são denominados de coortes. Outra compara a presença do fator etiológico em estudo em grupos de atingidos e não atingidos pela patologia. Quando a verificação da presença do fator etiológico é buscada em dados que antecederam a seleção dos grupos, denominamos estes estudos de caso-controle. No entanto, quando o aspecto tempo não é levado em consideração, a presença do fator etiológico e da patologia são medidos em um mesmo momento. A esta conduta dá-se o nome de estudo seccional ou transversal.

\begin{tabular}{|c|c|c|c|}
\hline \multicolumn{3}{|c|}{ Tabela 1 - Grupos populacionais em estudo. } \\
\hline Fator Etiológico & \multicolumn{3}{|c|}{ Efeito (patologia) } \\
\hline Hipótese & Com DTM & Sem DTM & Total \\
\hline Com má oclusão & A & B & A + B \\
\hline Sem má oclusão & C & D & C + D \\
\hline Total & A + C & B + D & $($ A + B +C +D) \\
\hline
\end{tabular}

\section{CONCEITOS E DEFINIÇÕES DAS DESORDENS TEMPOROMANDIBULARES}

O termo DTM, proposto por Bell ${ }^{4}$ para designar as desordens musculoesqueléticas do sistema mastigatório, embora amplamente aceito, engloba condições que agrupadas, não apresentam etiologia ou justificativa biológica comum. Tais desordens caracterizam um grupo heterogêneo de problemas de saúde cujos sinais e sintomas sobrepõem-se mas não são, necessariamente, idênticos.

Assim, critérios específicos de inclusão e exclusão para o diagnóstico destas desordens são fundamentais e devem ser testados para determinação de sua validade. No entanto, do ponto de vista clínico, não é importante ampliar extensivamente a divisão de subgrupos se todas as desordens dentro de um mesmo subgrupo podem ser controladas pelos mesmos procedimentos terapêuticos.

Atualmente o RDC/TMD (Research diagnostic criteria for temporomandibular desorders $)^{6}$ oferece a melhor classificação para agrupar as DTM. Neste sistema as desordens musculares, os desarranjos do disco articular e as condições dolorosas e degenerativas da ATM são classificadas como grupos diagnósticos distintos definidos por critérios específicos, embora não mutuamente excludentes. No entanto, uma das possíveis limitações deste sistema diagnóstico é a aparente inter-relação observada entre as diferentes categorias diagnósticas. De acordo com Turk ${ }^{14}$, em estudo com pacientes com DTM classificados pelo RDC/TMD, 60\% dos pacientes receberam mais de um diagnóstico, tendo 35\% recebido 3 ou mais diagnósticos. Desta forma a existência e a extensão da relação entre os diferentes subgrupos das DTM abre um amplo campo para a investigação científica.

\section{CONSIDERAÇÕES GERAIS SOBRE A ANÁLISE DA RELAÇÃO ENTRE MÁ OCLUSÃO E DTM}

Teoricamente todas as patologias podem ter etiologia multifatorial, no entanto, a abordagem dos conceitos de fatores etiológicos necessários para o aparecimento de uma determinada 
condição e aqueles por si só suficientes deve ser realizada com cautela. Um fator etiológico é necessário se a patologia em questão nunca puder acontecer na ausência deste fator. Já um fator etiológico é considerado suficiente quando sua presença sempre levar ao surgimento de determinada patologia. É possível, por exemplo, que um fator etiológico suficiente não seja um fator considerado necessário. Os fatores etiológicos para desordens dolorosas crônicas, como por exemplo as DTM, usualmente não podem ser considerados isoladamente necessários ou suficientes, e sim como parte de um conjunto suficiente de causas ${ }^{12}$.

Os estudos epidemiológicos a cerca da etiologia das DTM freqüentemente apresentam problemas metodológicos. Tais problemas podem ser justificados em parte pelo fato dos indivíduos analisados não poderem ser isolados dos demais fatores confudentes de uma maneira tão clara como na pesquisa experimental clássica ${ }^{1}$. Os estudos que relacionam as más oclusões e as DTM usualmente o fazem por meio de abordagens causais, diagnósticas ou terapêuticas. No entanto, conclusões a respeito de um tipo de estudo não são necessariamente aplicáveis a outros. Um fator pode ser etiológico sem nenhum papel diagnóstico ou altamente predictivo sem ser um fator de risco para a patologia em questão.

\section{ABORDAGEM CAUSAL EM ESTUDOS OBSERVACIONAIS}

Entende-se como causalidade a situação em que um fator contribui para o aparecimento de uma patologia e a remoção deste fator altera a freqüência ou a intensidade deste resultado. Dentre as estratégias observacionais, os estudos de coorte representam a evidência mais forte para a avaliação de causalidade. Contudo, o acompanhamento de pacientes por um longo período de tempo torna estes estudos demorados e dispendiosos, diminuindo consideravelmente o número publicações com estas características. Com maior freqüência encontramos estudos de caso-controle e estudos transversais por serem mais rápidos, práticos e menos dispendiosos, porém sujeitos a problemas metodológicos que dificultam o estabelecimento de relações de causalidade.

Estudos seccionais realizados por Ross Tallents e seus colaboradores, pertencentes ao Centro Dental de Eastman, Universidade de Rochester, relacionaram a oclusão dentária com o desenvolvimento de DTM intra-articulares. Um de seus residentes, Julian Kahn ${ }^{8}$, publicou em 1998 um estudo que avaliou a relação entre a sobremordida e a sobressaliência dos dentes anteriores de 82 voluntários assintomáticos e 263 pacientes com sintomatologia intra-articular espontânea ou durante os movimentos mandibulares. A avaliação por imagens de ressonância magnética demonstrou que $67 \%(n=55)$ dos voluntários assintomáticos apresentavam uma relação normal do complexo côndilo-disco-fossa articular e 33\% (n=27) apresentavam o disco articular deslocado. Entre os pacientes sintomáticos, $84 \%(\mathrm{n}=221)$ apresentavam deslocamento do disco articular e 16\% (n=42) apresentavam articulações normais. Quando comparamos a presença de transpasse horizontal maior ou igual a $4 \mathrm{~mm}$ entre indivíduos sintomáticos com deslocamento do disco articular e indivíduos assintomáticos com articulações normais, encontramos uma probabilidade cerca de 7 vezes maior (razão de chance $=7.749, \mathrm{p}<0.001$ ) para o desenvolvimento de dor intra-articular e deslocamento do disco articular.

Estes resultados demonstram que alterações morfológicas oclusais, especificamente neste caso o aumento do transpasse horizontal, podem aumentar o risco do desenvolvimento de subgrupos específicos de DTM como os desarranjos internos do disco articular e a artralgia temporomandibular. Isto não sugere que estes pacientes necessitem de tratamento mas que tais fatores devem ser considerados quando procedimentos ortodônticos ou ortognáticos estiverem indicados por outras razões, como por exemplo a estética. 


\section{ABORDAGEM DIAGNÓSTICA EM ESTUDOS OBSERVACIONAIS}

Definimos como teste diagnóstico todo procedimento complementar que ajude a confirmar ou refutar um diagnóstico clínico. A validade de um teste descreve a proporção de todos os seus resultados verdadeiros, positivos ou negativos, e é definida pela comparação com uma referência considerada a que melhor diagnostica a condição em questão, denominada padrão-ouro. Considerar a presença de má oclusão como um fator positivo para o diagnóstico das DTM coloca o diagnóstico prévio destas desordens, realizado por exames clínicos e de imagem de validade comprovada, como o padrão-ouro. Desta maneira, o grau de validade encontrado pode ser considerado como uma medida indireta da relação entre as variáveis má oclusão e DTM.

Os estudos retrospectivos relacionando fatores oclusais e DTM com maior consistência metodológica e estatística podem ser atribuídos a Pullinger e Seligman, da Faculdade de Odontologia da Universidade da Califórnia, Los Angeles. Em artigo recentemente publicado ${ }^{11}$, as características oclusais em dois grupos de mulheres com desordens intra-articulares (1993, $n=257$ e 1998, $n=124)$, diferenciadas nos subgrupos deslocamento do disco e osteoartrose, foram comparadas com controles assintomáticos pareados por gênero e por idade (1993, n=51 e 1998, n=47) por meio da análise de regressão logística múltipla.

Segundo os resultados apresentados, pacientes com deslocamento do disco articular são melhor caracterizados por mordida cruzada posterior unilateral e longos desvios das posições de relação cêntrica (RC) para máxima intercuspidação habitual (MIH). Pacientes com osteoartrose foram mais consistentemente caracterizados por longos desvios de RC para MIH ( $>2 \mathrm{~mm}$ ), longos transpasses horizontais ( $>4 \mathrm{~mm}$ ) e transpasses verticais negativos. Riscos aumentados para estas desordens (razão de chance $>2: 1$ ) foram predominantemente associados com variações extremas das condições oclusais, porém a prevalência destas foi bastante baixa na população estudada.

A discrepância entre RC e $\mathrm{MIH}$, mesmo não caracterizando uma má oclusão morfológica isoladamente, foi incluída na avaliação da ocorrência das DTM de origem articular por ser fundamental na definição da posição mandibular e, conseqüentemente, capaz de alterar a classificação morfológica das más oclusões.

O aumento do risco de deslocamento do disco articular relacionado à presença de mordida cruzada posterior contribui para a indicação de tratamento precoce para esta má oclusão, assim como a possibilidade de desenvolvimento de assimetrias esqueléticas faciais, mesmo que temporárias ${ }^{10}$. No entanto, não podemos atribuir qualquer efeito terapêutico aos procedimentos de expansão rápida da maxila, por exemplo, na solução de deslocamentos do disco articular já estabelecidos.

Longos desvios entre RC e $\mathrm{MIH}$ (>2 mm) e longos transpasses horizontais ( $>4 \mathrm{~mm}$ ) estão predominantemente ligados a casos de osteoartrose com menor representação nos deslocamentos do disco, os quais, teoricamente, deveriam preceder o aparecimento de alterações degenerativas intraarticulares adaptativas. $\mathrm{O}$ dilema apresentado ao clínico é onde estas situações são fatores etiológicos ou conseqüências de alterações degenerativas articulares.

\section{ABORDAGEM TERAPÊUTICA EM ESTUDOS EXPERIMENTAIS}

Embora conclusões sobre terapêutica e principalmente prevenção possam originar-se de estudos observacionais, uma maior consistência a cerca destas intervenções é alcançada por meio dos experimentos clínicos, preferencialmente aleatórios e controlados, pois permitem um maior controle sobre as variáveis confundentes. $\mathrm{O}$ experimento clínico examina a relação entre a intervenção e os resultados observados em uma coorte acompanhada por um determinado período de tempo.

As revisões sistemáticas de experimentos clínicos 
constituem a melhor evidência disponível para abordagens terapêuticas, pois reúnem, por meio de metodologia clara e reprodutível, os resultados de vários experimentos clínicos, alcançando objetivos bem definidos. Uma vez que a realização de intervenções visando a inclusão de fatores de má oclusão em indivíduos sadios não é eticamente plausível, a maior parte dos experimentos realizados utiliza o ajuste da oclusão, por exemplo, a eliminação e discrepâncias de RC para MIH, em pacientes com DTM.

Tsukiyama e colaboradores ${ }^{13}$ conduziram uma revisão sistemática da literatura publicada na língua inglesa envolvendo experimentos clínicos de ajuste oclusal para o tratamento das DTM. Foram encontrados 6 experimentos com o total de 219 pacientes distribuídos em 9 publicações avaliando o sucesso da terapia de ajuste oclusal no tratamento de pacientes com DTM. De uma maneira geral, estas intervenções não demonstraram eficácia terapêutica elevada quando comparados com seus controles. No entanto, as definições de DTM utilizadas pelos estudos revisados não incluem a classificação em subgrupos específicos, como, por exemplo, as artralgias temporomandibulares, osteoartroses e os desarranjos do disco articular, o que torna os resultados obtidos pouco conclusivos.

Um segundo tipo de experimento tem abordagem preventiva e pode ser conduzido pela realização de ajustes oclusais profiláticos e fictícios em pacientes sadios, os quais são acompanhados por um determinado período de tempo para a verificação da freqüência do desenvolvimento de DTM. Apenas um grupo de pesquisadores, liderados por Penti Kirveskari, tem conduzido este tipo de experimento.

Em artigo publicado em 1998, Kirveskari e outros $^{9}$ apresentaram os resultados de um experimento clínico com 146 crianças e adolescentes saudáveis. Cerca da metade dos indivíduos ( $\mathrm{n}=$ 74) receberam ajuste oclusal para eliminação dos fatores de risco presumidos, incluindo as discrepâncias entre RC e MIH, e a outra parte ( $n$ = 72) recebeu ajustes simulados. Os ajustes foram repetidos a cada 6 meses por um período de 4 anos. O resultado esperado foi a incidência de DTM, operacionalmente definida como a procura por tratamento para os sintomas característicos destas desordens confirmados clinicamente. A incidência acumulativa encontrada foi de 9/67 para os controles e 1/60 para o grupo que sofreu o ajuste, o que significa um risco cerca de 8 vezes maior dos indivíduos não submetidos a ajustes oclusais desenvolverem DTM $(\mathrm{RR}=8.06, \mathrm{p}<0.019)$.

No entanto, o número de abandonos do experimento foi consideravelmente maior $(n=14)$ nos indivíduos submetidos ao ajuste oclusal do que nos controles $(\mathrm{n}=5)$. Tal fato põe em dúvida o desconhecimento por parte dos pacientes a respeito do grupo experimental do qual participavam e compromete a definição da incidência de DTM pela busca espontânea por tratamento. Quando comparamos a incidência de ruídos articulares, observados ou relatados pelos pacientes durante as visitas periódicas, as medidas de risco decrescem a níveis não significantes ( risco relativo $=0,87$ e risco relativo $=0,78$ ) .

Os resultados dos estudos acima citados não justificam o emprego de ajuste oclusal profilático na prevenção das DTM, pois sua eficácia não é conhecida. Tais procedimentos são provavelmente inefetivos na grande maioria dos casos, devido à presumível influência de outros fatores não oclusais no modelo destas desordens.

\section{CONCLUSÕES}

A definição dos possíveis fatores etiológicos relacionados a subgrupos específicos de DTM é fundamental para que o papel das maloclusões no desenvolvimento destas desordens, embora pareça pequeno quando baseado nas evidências disponíveis, não seja subestimado.

Pode ser útil a caracterização de uma oclusão normal como aquela associada como o menor risco para o desenvolvimento de problemas de DTM, mas é provavelmente inapropriada a aplicação destes parâmetros para reverter um problema 
intra-capsular já estabelecido.

O conceito de uma oclusão de baixo fator de risco implicaria em um pequeno desvio entre $\mathrm{RC}$ e $\mathrm{MIH}$, pequeno transpasse horizontal, transpasse vertical positivo e ausência de mordida cruzada posterior. Este conceito é compatível com o conceito de oclusão normal defendido por décadas, embora uma variação do normal ao invés de um critério absoluto deva ser permitido.

Embora provavelmente seja prudente estabelecer metas morfológicas terapêuticas que busquem o que é observado em oclusões não tratadas julgadas normais ou ideais ${ }^{3}$, o estabelecimento de uma oclusão que alcance todos os critérios gnatológicos, por meio de tratamento ortodôntico, talvez seja impossível e provavelmente desnecessário.

\title{
Epidemiological basis for the analysis of morphologic malocclusion as a risk factor for the development of temporomandibular joint disorders
}

\begin{abstract}
Among the researches undertaken in epidemiology, a peculiar group of pathologies present either unknown or not totally understood origins. Temporomandibular disorders (TMD) can be classified in this subgroup. Three basic observational strategies have been used to approach the etiologic role of malocclusion in TMD development, inside the epidemiological repertoire. They are sectional, case-control and cohort studies. Some clinical trials are conducted based on the removal of the suspect etiologic factor. Based on the literature reviewed in terms of the methodology applied in the selected studies, we can infer that: the definition of possible etiological factors related to specific sub-groups of TMD is primordial not to underestimate the role of malocclusion in the development of this kind of disorders; the characterization of a normal occlusion like the one associated with the lower risk for TMD problems development can be useful, but the application of these parameters will probably result inappropriate for the resolution of a joint problem which is already established; the concept of lower risk occlusion would involve a small slide discrepancy between RCP and ICP, small incisal overlap, positive overbite and no posterior crossbite. This concept is similar to the concept of normal occlusion supported for decades, although a deviation from the normal criterion instead of an absolute criterion must be tolerated; although it may be prudent to establish therapeutic morphologic goals that seek what is observed in untreated occlusions deemed normal or ideal, the establishment of an occlusion that reach all these gnathologic criteria, by means of orthodontic treatment, might be either impossible or probably unnecessary.
\end{abstract}

Key words: Malocclusion. Temporomandibular disorders. Epidemiology. 


\section{REFERÊNCIAS}

1. ALANEN, P. J.; KIRVESKARI, P. K. Disorders in TMJ research. J Craniomandib Disord, Lombard, v. 67, p.113-120, Apr. 1990.

2. ALMEIDA FILHO, N.; ROUQUA, YROL, M. Z. Introdução à epidemiologia moderna 2. ed. Belo Horizonte: Coopmed-ApceAbrasco, 1992.

3. ANDREWS, L. F. The six keys to normal occlusion. Am J Orthod, St. Louis, v.62, no.3, p.296-309, Sept. 1972.

4. BELL, W. E. Clinical management of temporomandibular disorders. Chicago: Year Book Medical, 1982.

5. CAPRA, F. O modelo biomédico. In: CAPRA, F. O ponto de mutação. São Paulo: Cultrix, 1992. p.116-155.

6. DWORKIN, S. F.; LERESCHE, L. Research diagnostic criteria for temporomandibular disorders: review, criteria, examinations and specifications, critique. J Craniomandib Disord, Lombard, v. 6, no. 4, p.301-355, Apr. 1992.

7. GOLDESTEIN, B. H. Temporomandibular disorders: a review of current understanding. Oral Surg Oral Med Oral Pathol Oral Radiol Endod, St. Louis, v. 88, no. 4, p.379-385, Oct. 1999.

8. KAHN, J.; TALLENTS, R. H.; KATZBERG, R. W.; ROSS, M. E. MURPHY, W. C. Association between dental occlusal variables and intraarticular temporomandibular joint disorders: horizontal and vertical overlap. J Prosthet Dent, St. Louis, v. 79, no. 6, p. 658-662, June 1998.
9. KIVERSKARI, P.; JAMSA, T.; ALANEN, P. Occlusal adjustment and the incidence of demand for temporomandibular disorder treatment. J Prosthet Dent, St. Louis, v.79, p. 433-438, Apr. 1998.

10. PINTO, A. S.; BUSCHANG, P. H.; THROCKMORTON, G. S.; CHEN, P. Morphological and positional asymmetries of young children with functional unilateral posterior crossbite. Am J Orthod Dentofacial Orthop, St. Louis, v.120, no. 5, p. 513-520, Nov. 2001.

11. PULLINGER, A. G.; SELIGMAN, D. A. Quantification and validation of predictive values of occlusal variables in temporomandibular disorders using a multifactorial analysis. J Prosthet Dent, St. Louis, v. 83, no.1, p. 66-75, Jan. 2000

12. ROTHMAN, K. J. Modern epidemiology. Boston: Little Brown, 1986.

13. TSUKIYAMA, Y: BABA, K. CLARK, G. T. An evidence-based assessment of occlusal adjustment as a treatment for temporomandibular disorders. J Prosthet Dent, St. Louis, v.86, no.1, p. 57-66, July 2001.

14. TURK, D. C. Psychosocial and behavioral assessment of patients with temporomandibular disorders: diagnostic and treatment implications. Oral Surg Oral Med Oral Pathol Oral Radiol Endod, St. Louis, v. 83, no.1, p. 65-71, Jan. 1997.
Endereço para correspondência

Ricardo de Souza Tesch

Praça Dr. Sá Earp, 22 - sala 602

Centro - CEP: 25655-111

Petrópolis - RJ

E-mail: ricardot@compuland.com.br 